\title{
Electro-optic co-design for next generation silicon optical transmitters
}

Breyne, L., Lambrecht, J., Vanhoecke, M., Ossieur, P., Roelkens, G., et al.

L. Breyne, J. Lambrecht, M. Vanhoecke, P. Ossieur, G. Roelkens, J. Bauwelinck, X. Yin, "Electro-optic co-design for next generation silicon optical transmitters," Proc. SPIE 11880, Emerging Applications in Silicon Photonics II, 118800L (6 October 2021); doi: 10.1117/12.2601596

SPIE. Event: SPIE Photonex, 2021, Glasgow, Scotland, United Kingdom 


\title{
Electro-optic co-design for next generation silicon optical transmitters \\ L. Breyne*a , J. Lambrecht ${ }^{\mathrm{a}}$, M. Vanhoecke ${ }^{\mathrm{a}}$, P. Ossieur ${ }^{\mathrm{a}}$, G. Roelkens ${ }^{\mathrm{b}}$, J. Bauwelinck ${ }^{\mathrm{a}}$ and X. Yin ${ }^{\mathrm{a}}$ ${ }^{a}$ IDLab, imec - Ghent University, Technologiepark 126, Ghent 9000, Belgium; bPhotonics Research Group. Ghent University, Technologiepark 126, Ghent 9000, Belgium;
}

\begin{abstract}
This paper discusses our progress on high-speed optical transmitters for next generation intra-datacenter interconnects. Silicon integrated photonic systems have a key role to play in this evolution by allowing compact, fast, innovative and cost-effective devices to be manufactured in large volumes. Especially silicon Mach-Zehnder modulators are a very attractive candidate: they are easy to manufacture, easy to use and support both intensity as well as coherent modulation. Key to the next-generation optical transmitter is not only the very high datarates, but also the very small form-factor and low power consumption. This requires leveraging electro-optic co-design of driver electronics and optical modulators.
\end{abstract}

Keywords: Silicon Photonics, Mach-Zehnder modulators (MZMs), integrated driver electronics, digital-to-analog converters (DACs), electro-optic co-design, equalization, finite impulse response (FIR) filters, slow-wave designs

\section{INTRODUCTION}

Last decades, the internet has experienced explosive growth fuelled by the emergence of newer and more powerful applications and technologies ${ }^{1}$. The internet of things, virtual and augmented reality, 5G, cloud computing and many others are presenting themselves to continue this fast-paced growth. Such applications put datacenters, and the optical interconnections inside, under high pressure. Academia and industry are racing to develop optical interconnects that meet the ever-increasing demand for higher datarates. Most recently approved standards focus on 400 Gbps interconnects, using 4 lanes of 53 Gbaud PAM42 ${ }^{2}$. Near future interconnects aim at $800 \mathrm{Gbps}^{3}$, initially over 8 and later over 4 lanes, and 1.6 Tbps datarates.

Critical to these optical interconnects are very fast, low power and compact transceivers. Focussing on the transmitter, this can be achieved by thorough co-design of both the driving electronics and the optical modulator. Silicon photonics is a key enabler for such transmitters, as silicon optical circuits are compact, low-loss and can be manufactured with highyield and in high-volumes. Several promising PAM4 transmitters have been demonstrated ${ }^{4,5,6,7,8}$. Often microring modulators (MRMs) or electro-absorption modulators (EAMs) are chosen over Mach-Zehnder modulators (MZMs) due to their very small footprint and very low power consumption ${ }^{4,7,8}$. However MRMs are quite difficult to bias due to their high wavelength dependency. On the other hand, EAMs require additional materials (like Germanium) and only operate in the optical O- or C-band. MZMs are easy to bias, are optically broadband, do not require additional materials and are more suited for coherent optical communications. Coherent communications is being developed for inter-datacenter interconnections and is expected to be used for intra-datacenter interconnects in the future ${ }^{9}$. Thus silicon MZMs are a very popular and future proof choice as modulators for datacenter interconnects.

As the electro-optic effect in silicon is relatively limited compared to e.g III-V materials ${ }^{10}$, the modulator length is in the order of several millimeters. Thus for most high-speed designs, traveling-wave (TW) structures are used to reach very high bandwidths. By optimizing the PN junction itself ${ }^{11}$, the diode configuration ${ }^{12}$, the electrodes ${ }^{12,13}$ and the architecture of the modulator ${ }^{14}$, silicon MZMs are able to keep up with the continuous demand for more bandwidth.

In the first part of this work, we discuss our work on electrode segmentation to realise a 50 Gbaud PAM4 electro-optical digital-to-analog converter (EO-DAC). By splitting the long TW design into two TW segments, higher bandwidths can be obtained while at the same time a 2-bit DAC functionality is implemented. So power-hungry PAM4 driving electronics can be replaced by more-power efficient limiting drivers. We report 50 and 53 Gbaud PAM4 generation with an excellent power efficiency of 3.9 and $3.6 \mathrm{pJ} / \mathrm{bit}^{15}$. In the second part, our progress on a novel modulator design technique is presented. By well chosen adjustment to the optical routing of a slow-wave modulator, a finite impulse response (FIR) filter can be embedded directly inside the modulator. This method of equalization can be used to extend the modulator bandwidth or even take over (part) of the electrical equalization used in optical communication links ${ }^{16}$.

*Laurens.Breyne@imec.be; www.imec-int.com,www.idlab.ugent.be

Emerging Applications in Silicon Photonics II, edited by Callum G. Littlejohns, Marc Sorel, Proc. of SPIE Vol. 11880, 118800L · @ $2021 \mathrm{SPIE} \cdot$ CCC code: 0277-786X/21/\$21 · doi: 10.1117/12.2601596 


\section{50 GBAUD PAM4 OPTICAL TRANSMITTER}

\subsection{Introduction}

In traveling-wave modulators, a trade-off between $\mathrm{V}_{\pi}$ and bandwidth exists: a longer device exhibits a lower $\mathrm{V}_{\pi}$ at the cost of bandwidth. This is caused by the microwave attenuation of the driving signal on the electrodes, which is proportional to the length of the device. The authors of ${ }^{14}$ show that this trade-off can be circumvented by splitting the long phase shifter into two shorter phase shifters (which have a combined length equal to the long phase shifter), the same $\mathrm{V}_{\pi}$ is achieved but each segment has a higher bandwidth. One can drive both segments with the same signal, e.g. the desired 50G PAM4 signal, or one can drive the segments with the MSB and LSB of the PAM4 signal to convert two NRZ signals into a PAM4 signal. If both MSB and LSB signals have the same swing, then the MSB segment should be twice as long as the LSB segment to realize a correct conversion. Besides the higher bandwidth, this solution also allows the use of more power efficient limiting driving electronics instead of power hungry linear electronics.

\subsection{Driver-modulator interfacing}

A differential driver is preferred as it provides advantages in driving voltage, bandwidth, signal quality and inter-device crosstalk $^{17}$. A simplified connection diagram is shown in Fig. 1. DC-coupling is chosen over AC-coupling since DCcoupling allows the large DC-driver current to be split over $\mathrm{R}_{\mathrm{L}}$ and $\mathrm{R}_{\mathrm{T}}$. AC-coupling further complicates the integration due to the required series capacitors in the datapath. By using a series push-pull (SPP) phase shifter ${ }^{12}$ in the modulator, the PN junctions can be reverse biased through the bias line B (Fig. 1 (b)) and the voltage $\mathrm{V}_{\mathrm{B}}$ (Fig. 1 (a)). So the junction bias voltage is optimized independently from the DC-point of the driver. Lastly, by changing the voltage $\mathrm{V}_{\mathrm{S}}$ and $\mathrm{V}_{\mathrm{T}}$, the headroom in the driver can be adjusted to further optimize the performance and power consumption.
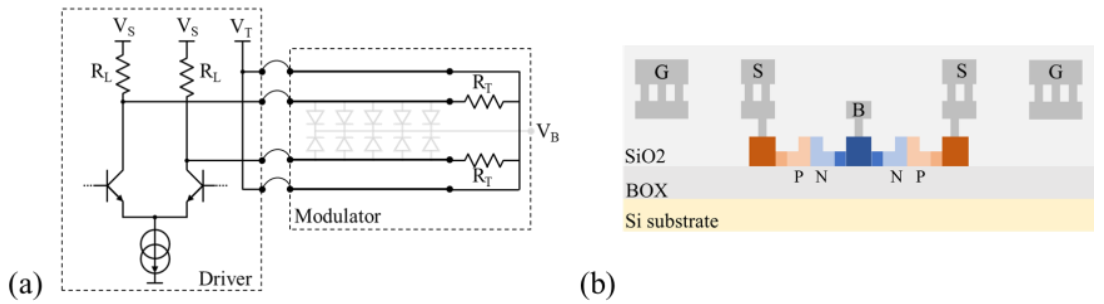

(b)

Figure 1. (a) Equivalent circuit of the modulator, simplified driver connection circuit and DC-coupled interface. (b) Cross section of the differential modulator with the series-push-pull phase shifters.

\subsection{Modulator design}

The modulators were designed on imec's iSiPP50G platform ${ }^{18}$. The default phase shifters from the PDK were used. First the cross-sectional dimensions of the electrode were optimized to minimize the microwave attenuation while also having a characteristic impedance as close as possible to $100 \Omega$ and achieving velocity matching between the electrical and optical signal. Second, based on requirements on the extinction ratio (ER) and using the driver swing $\left(2 \mathrm{~V}_{\mathrm{pp}}\right)$, the lengths of the MSB and LSB were chosen to be 1.2 and $2.25 \mathrm{~mm}$. The on-chip termination is done through $50 \Omega \mathrm{N}$-doped resistors. Lastly, some small optimizations to the cross-sectional dimensions of the electrodes were done to optimize the bandwidth. The final architecture of the modulator, operating in the C-band, is shown in Fig. 2 (a).

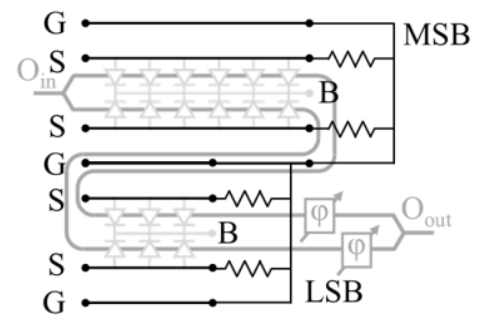

(a)

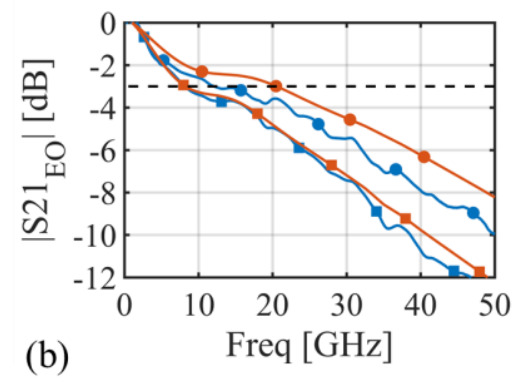

(b)

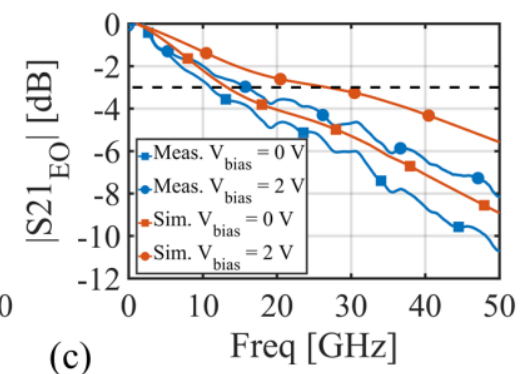

(c)

Figure 2. (a) Architecture of the full modulator. (b) and (c) simulated and measured electro-optical response of the MSB and LSB respectively at 0 and $2 \mathrm{~V}$ reverse bias. 
From the DC-measurements on the devices at a reverse bias of $2 \mathrm{~V}$, we found that the $\mathrm{V}_{\pi}$ of the MSB and LSB were respectively 9.8 and $18.4 \mathrm{~V}$ and the insertion loss is 3.8 and $2 \mathrm{~dB}$. So the combined $\mathrm{V}_{\pi}$ is $6.4 \mathrm{~V}$ and the combined phase shifter insertion loss is $5.8 \mathrm{~dB}$. The simulated and measured electro-optic responses are shown in Fig. 2 (b) and (c). Th EO 3dB bandwidth of the MSB and LSB is lower than expected 12.1 and $15.9 \mathrm{GHz}$. However, the driver is able to introduce peaking in its response to counteract this bandwidth limitation ${ }^{15}$. The main reason for this drop in bandwidth is the higher-than-expected termination which causes the fast roll-off at low frequencies.

\subsection{Experimental results}

Both the modulator and driver are assembled on a test PCB using wirebonding, see Fig. 3(a). The PCB features connectors to provide high-speed PRBS15 signals to the driver. The driver chip consists of four limiting channels, each capable of generating $56 \mathrm{Gbps} 2 \mathrm{~V}_{\mathrm{pp}}$ in a $100 \Omega^{15}$ differential load with a power consumption of approx. 200 $\mathrm{mW} / \mathrm{channel}$, depending on the exact driver settings and supply voltage. The output stage delivers a binary drive signal, but is able to introduce a few $100 \mathrm{mV}$ of peaking to counteract potential bandwidth limitation in the modulator. The chip is manufactured in a $55 \mathrm{~nm}$ SiGe BiCMOS technology. Optical interfacing is done through fiber probes, while the MZM is configured using thermo-optic heaters. The quality of the PAM4 eyes is assessed through the TDECQ figure of merit ${ }^{15}$, the results for various rates are shown in Fig. 3 (b) to (d). The driver power supply $\mathrm{V}_{\mathrm{S}}$ is $2.75 \mathrm{~V}, \mathrm{~V}_{\mathrm{T}}$ is $3.1 \mathrm{~V}$ and the reverse bias voltage over the PN junctions is $3.1 \mathrm{~V}$ to maximize the bandwidth. The driver is also configured to induce some peaking at the output. The swing of the channel driving the LSB is slightly optimized to obtain perfectly equidistant PAM4 levels (RLM>0.92 in all three measurements). The extinction ratio measured on the outer levels is approximately $4.5 \mathrm{~dB}$. Most standards ${ }^{2}$ require a TDECQ below $3.4 \mathrm{~dB}$, which is satisfied up to 50 Gbaud. By redesigning the modulator with lower on-chip terminations, the bandwidth can be extended and we expect to also satisfy the TDECQ specification at 53 Gbaud. At 40G, the MSB and LSB channels are consuming 199 and $175 \mathrm{~mW}$. While at 50 and 53 Gbaud, the MSB and LSB channels are consuming 201 and $184 \mathrm{~mW}$ respectively, resulting in an excellent power efficiency of $3.9 \mathrm{pJ} / \mathrm{bit}$ and $3.6 \mathrm{pJ} / \mathrm{bit}$ at 50 and 53 Gbaud PAM4

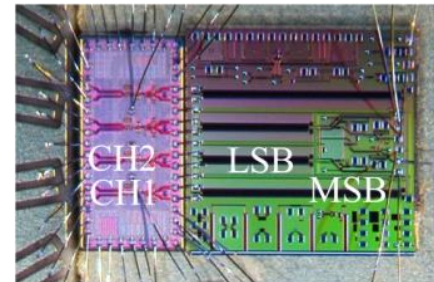

(a)

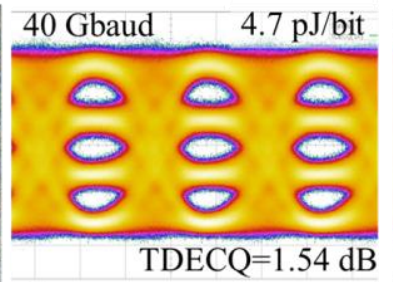

(b)

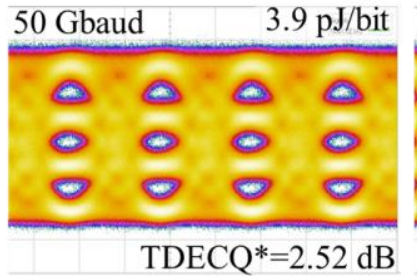

(c)

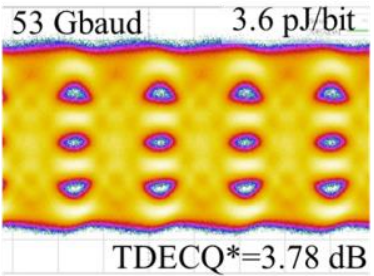

(d)

Figure 3. (a) Micrograph of the driver and modulator assembled on a PCB. (b-d) generated optical PAM4 signals after TDECQ processing.

\section{ELECTRO-OPTICAL FREQUENCY RESPONSE SHAPING}

\subsection{Introduction}

In this second part, we will shift our focus towards optimizing the bandwidth of the modulator itself. Typical high-speed silicon MZM designs using traveling-wave electrodes require close attention to both the phase shifters as well as the electrodes. Due to the loading of the phase shifter on the transmission line electrodes, additional microwave losses are introduced resulting in a decreased bandwidth. Using slow-wave transmission lines ${ }^{19}$, the phase shifters are divided into smaller, isolated segments which are periodically connected to the electrodes, see Fig. 4 (a). Qualitatively, one can state that this allows to 'tune' the effective load and optimize the characteristic impedance, electro-optic velocity matching and minimize the microwave attenuation. The slow-wave modulator is shown to have superior high-speed performance ${ }^{19,20}$ compared to its continuous counterpart.

In this section, we show that using careful analysis of the slow-wave structure, a finite-impulse-response filter structure can be identified. Using minor adjustments to the optical routing between phase shifting segments, it becomes possible to alter the electro-optic frequency response to the designer's needs. So, one can introduce peaking to improve the bandwidth of the modulator, or over-compensate in the modulator in order to alleviate bandwidth limitations in for example the driving electronics. We apply the technique to modulators and show the advantage using a transmission experiment. 


\subsection{Embedding FIR filtering in slow-wave modulators}

A standard slow-wave modulator design is shown in Fig 4 (a). Similar to Section 2, a differential modulator with seriespush-pull phase shifters is chosen for optimal integration with the driving electronics. A full analytical description of this structure is given in ${ }^{19,20}$. In order to gain most insights, we derive a simplified block diagram description of one modulator arm. First, the transmission line sections between adjacent phase shifters can be simplified to a delay $\mathrm{T}_{\mathrm{E}}$. The same can be done with the optical waveguides between two segments, those can be simplified to a delay $\mathrm{T}_{\mathrm{O}}$. The phase shifters can be replaced by multipliers with a certain phase shift PS at their output. In the optical domain, the phase shift induced by one segment can be added to the phase shift of the optical signal applied to that segment. As such, the equivalent block diagram in Fig. 4 (b) is obtained.

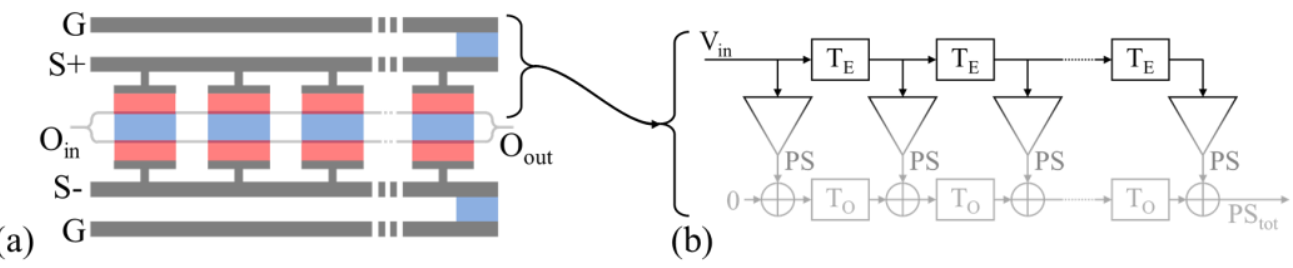

Figure 4. (a) Layout of a slow-wave modulator with differential electrodes. (b) Simplified block diagram of the top arm of the differential modulator in (a).

The simplified block diagram shows great similarity with the distributed implementation of a FIR filter. In a typical modulator design, the electrical and optical wave velocities are matched, such that $\mathrm{T}_{\mathrm{E}}$ and $\mathrm{T}_{\mathrm{O}}$ are equal. As a result, the signal from $V_{\text {in }}$ to $\mathrm{PS}_{\text {tot }}$ experiences the same delay through all phase shifters. So we can omit all $\mathrm{T}_{\mathrm{E}}$ and $\mathrm{T}_{\mathrm{O}}$ resulting in $\mathrm{PS}_{\mathrm{tot}}=\mathrm{N}$.PS with $\mathrm{N}$ the number of segments. By introducing an additional optical delay line and an optical crossing between two segments, a FIR filter can be implemented, see Fig. 5.

(a)

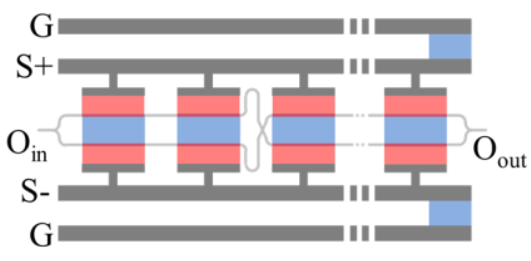

(b)

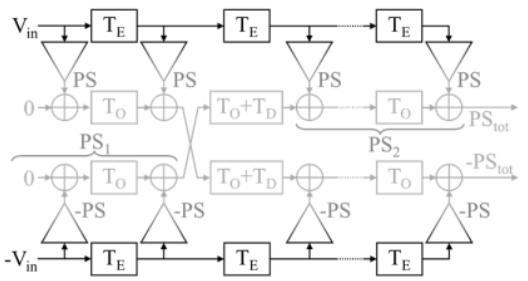

Figure 5. (a) Layout of a slow-wave modulator with an optical crossing and delay line between the $2^{\text {nd }}$ and $3^{\text {rd }}$ segment. (b) Simplified block diagram of the modulator (a).

The optical crossing and delay line implemented in Fig 5 (a) has two effects on the block diagram of Fig. 5 (b). First there is an additional delay $T_{D}$ added to $T_{O}$. Second, the output of the top arm, which normally only consists of positive phase shift contributions, now also contains negative phase shift contributions. Note that the modulator is driven in a push-pull regime (differential signalling). As $\mathrm{T}_{\mathrm{E}}$ and $\mathrm{T}_{\mathrm{O}}$ are still equal, we omit $\mathrm{T}_{\mathrm{E}}$ and $\mathrm{T}_{\mathrm{O}}$ in the modulator, which yields $\mathrm{PS}_{1}=-2 \mathrm{PS}$ and $\mathrm{PS}_{2}=(\mathrm{N}-2) \mathrm{PS}$. Continuing, the frequency response of $\mathrm{PS}_{\mathrm{tot}}$ is then $\mathrm{PS}_{\mathrm{tot}}=\mathrm{PS}_{1} \cdot \exp \left(-\mathrm{j} 2 \pi \mathrm{f} \mathrm{T}_{\mathrm{D}}\right)+\mathrm{PS}_{2}$, with $\mathrm{f}$ the microwave frequency and $\exp \left(-\mathrm{j} 2 \pi f \mathrm{~T}_{\mathrm{D}}\right)$ is the delay. Substituting $\mathrm{PS}_{1}, \mathrm{PS}_{2}$ and $\mathrm{z}^{-1}=\exp \left(-\mathrm{j} 2 \pi f \mathrm{~T}_{\mathrm{D}}\right)$, we find the $\mathrm{z}$-domain representation of the filter: $\mathrm{PS}_{\mathrm{tot}}=-2 \mathrm{PS} . \mathrm{z}^{-1}+(\mathrm{N}-2) \mathrm{PS}$. At DC, $\mathrm{PS}_{\mathrm{tot}}=(\mathrm{N}-4) \mathrm{PS}$ while at $\mathrm{f}=1 /\left(2 \mathrm{~T}_{\mathrm{D}}\right), \mathrm{PS}_{\text {tot }}$ is N.PS. Thus the FIR filter introduces peaking at high frequencies which can be used to extend the bandwidth of the modulator. By combining multiple crossings and/or delay lines at different positions in the modulator, more complicated transfer functions can be realized. The technique allows to tailor the electro-optic response of the modulator to the designer's needs by only changing the optical routing. No changes to the electrical part of the modulator or the phase shifters themselves are required. Note that the bandwidth extension comes at a cost: introducing peaking lowers the modulation efficiency. However, this cost is inherent to transmit side equalization. The above description shows well how the technique works, however, a full analytical solution including all transmission line effects can be found $\mathrm{in}^{16}$.

\subsection{Experimental results}

In order to verify the technique, two C-band devices were manufactured on imec's iSiPP50G platform ${ }^{18}$. A slow-wave modulator without adjustments and a modulator where optical delay lines were inserted between the $3^{\text {rd }} / 4^{\text {th }}$ and $4^{\text {th }} / 5^{\text {th }}$ 
segments and a crossing between the $3^{\text {rd }} / 4^{\text {th }}$ segments. Each modulator had 10 segments of 175 um with a pitch of 250 $\mathrm{um}$. The shaped modulator transfer function is thus $\mathrm{PS}_{\mathrm{tot}}=6 \mathrm{PS}+\mathrm{PS} \cdot \mathrm{z}^{-1}-3 \mathrm{PS} \cdot \mathrm{z}^{-2}$, the delay line introduces $7 \mathrm{ps}$ of delay. The layouts of both devices are shown in Fig. 6 (a). The RF input signals are applied to the GSSG bondpads at the left, $2 \times 50 \Omega$ terminations are provided on-chip. Optical coupling is done through fiber probes and grating couplers.

(a)
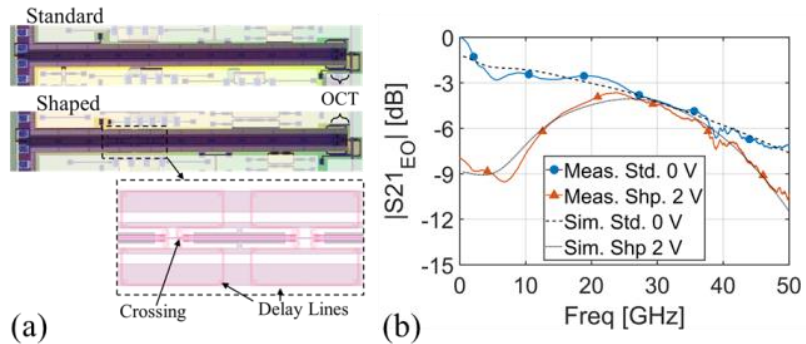

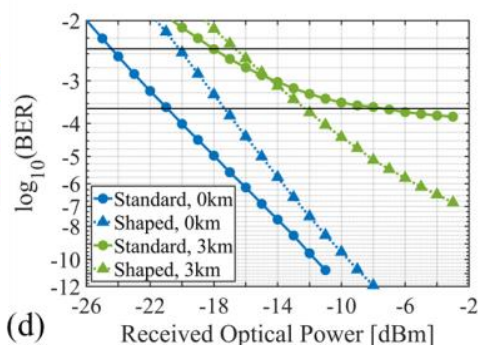

(c)

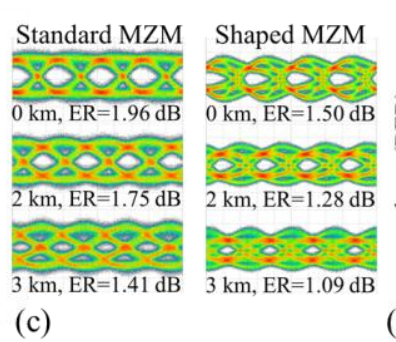

(d)

Figure 6. (a) Micrograph of both modulators, the inset of the crossing and delay. (b) Measured and simulated electrooptic transfer functions, normalized for the standard modulator at DC. (c) Measured eye diagrams of $56 \mathrm{Gbps}$ NRZ data transmission over 0,2 and $3 \mathrm{~km}$ fiber transmission in the C-band. (d) BER curves at 0 and $3 \mathrm{~km}$ as a function of average received power.

From the DC-measurements, we find that the $\mathrm{V}_{\pi}$ of the standard and shaped modulator is 11.8 and $29.6 \mathrm{~V}$ respectively for a reverse bias of $1 \mathrm{~V}$. The high $\mathrm{V}_{\pi}$ of the standard modulator comes from the fact that the modulator is relative short (only $1.75 \mathrm{~mm}$ total phase shift length). From the transfer function of the shaped modulator, we find at DC that PS only 4 PS, thus 2.5 times less than the standard modulator (10 PS). As a result, the $\mathrm{V}_{\pi}$ is 2.5 times higher. The insertion loss is 2.6 and $3.1 \mathrm{~dB}$ respectively for the standard and shaped modulator at $1 \mathrm{~V}$ reverse bias. The $0.5 \mathrm{~dB}$ excess loss from the shaped modulator stems from the two $500 \mathrm{um}$ long optical delay lines and the optical crossing. The measured and simulated electro-optic responses are shown in Fig. 6 (b). The measurements were done using a VNA and a 70 GHz reference photodiode. As can be observed, the simulations and measurements coincide very well. The 8 dB lower response for the shaped modulator again comes from the transfer function (4/10 is $-8 \mathrm{~dB})$. Lastly, both modulators were used in a $56 \mathrm{Gbps}$ NRZ IM/DD transmission experiment at $1550 \mathrm{~nm}$ over 0,2 and $3 \mathrm{~km}$ SSMF fiber. Due to chromatic dispersion, a bandwidth limitation will be introduced causing distortion. The modulators were driven with a $4 \mathrm{Vpp}$ signal. As can be seen in Fig. 6 (c), the shaped modulator has slightly smaller eyes at $0 \mathrm{~km}$. But after $3 \mathrm{~km}$, the shaped modulator has cleaner eyes due to the peaking. This is also confirmed the BER measurements in Fig. 6 (d). At 0 km, no bandwidth limitation is present and the standard modulator achieves a better BER. However, after $3 \mathrm{~km}$ and at sufficiently high receive power, the bandwidth limitation is dominant and the shaped modulator achieves a better BER.

\section{CONCLUSION}

In this invited paper, we have shown a segmented silicon traveling-wave silicon MZM integrated together with a highspeed SiGe BiCMOS driver. Using this 2-bit electro-optical DAC, we were able to generate 50 and 53 Gbaud PAM4 with an ER of $4.5 \mathrm{~dB}$ and a TDECQ of $2.78 \mathrm{~dB}$ and $3.78 \mathrm{~dB}$. An excellent power efficiency of 3.9 and $3.6 \mathrm{pJ} / \mathrm{bit}$ respectively was obtained. Next, we demonstrated a novel technique to optimize the electro-optic frequency response of a slow-wave MZM by changing the optical routing between segments. This technique allows to increase the device bandwidth or induce peaking to alleviate other bandwidth limitations in the link. So a cost, area and power efficient way of transmitter side equalization is obtained. With our work, we have shown the potential of silicon photonic transmitters towards future generations short-reach optical interconnects.

\section{ACKNOWLEDGMENTS}

This work was supported in part by the EU-funded H2020 Projects PICTURE (grant no. 780930) and the EU ERC Grant ATTO (grant no. 695495). This work was also supported by the Special Research Fund (BOF) of Ghent University and the Research Foundation Flanders (FWO). 


\section{REFERENCES}

[1] P. J. Winzer and D. T. Neilson, "From Scaling Disparities to Integrated Parallelism: A Decathlon for a Decade," Journal of Lightwave Technology, vol. 35, no. 5, pp. 1099-1115, 2017.

[2] "IEEE P802.3bs $200 \mathrm{~Gb} / \mathrm{s}$ and $400 \mathrm{~Gb} / \mathrm{s}$ Ethernet Task Force," Last accessed April 28, 2020.

[3] "800G PSM8 MSA," Last accessed January 15, 2021. Available on URL: https://www.800gmsa.com/

[4] H. Li et al., "A 112 GB/s PAM4 Transmitter with Silicon Photonics Microring Modulator and CMOS Driver," in 2019 Optical Fiber Communications Conference and Exposition (OFC), (2019), pp. 1-3.

[5] Y. Ma et al., "An all-silicon transmitter with co-designed modulator and DC-coupled driver," in 2019 Optical Fiber Communications Conference and Exposition (OFC), (2019), pp. 1-3.

[6] K. Zhong, J. Mo, R. Grzybowski, and A. P. T. Lau, "400 Gbps PAM-4 Signal Transmission Using a Monolithic Laser Integrated Silicon Photonics Transmitter," in 2019 Optical Fiber Communications Conference and Exposition (OFC), (2019), pp. 1-3.

[7] J. Verbist et al., "Real-time and DSP-free $128 \mathrm{~Gb} / \mathrm{s}$ PAM-4 link using a binary driven silicon photonic transmitter," J. Lightw. Technol., vol. 37, no. 2, pp. 274-280, 2019.

[8] S. Fathololoumi et al., "1.6 Tbps Silicon Photonics Integrated Circuit and 800 Gbps Photonic Engine for Switch CoPackaging Demonstration," in Journal of Lightwave Technology, vol. 39, no. 4, pp. 1155-1161, 15 Feb.15, 2021

[9] J. K. Perin, A. Shastri and J. M. Kahn, "Coherent Data Center Links," in Journal of Lightwave Technology, vol. 39, no. 3, pp. 730-741, 1 Feb.1, 2021.

[10] R. Soref and B. Bennett, "Electrooptical effects in silicon," IEEE J. Quantum Electron., vol. QE-23, no. 1, pp. 123129, Jan. 1987.

[11] D. Petousi et al., "Analysis of optical and electrical tradeoffs of traveling-wave depletion-type Si Mach-Zehnder modulators for high-speed operation," IEEE J. Sel. Top. Quantum Electron., vol. 21, no. 4, pp. 199-206, 2015.

[12] P. Dong, L. Chen, and Y. kai Chen, "High-speed low-voltage single-drive push-pull silicon Mach-Zehnder modulators," Opt. Express, vol. 20, no. 6, pp. 6163-6169, Mar. 2012.

[13] H. Yu and W. Bogaerts, "An equivalent circuit model of the traveling wave electrode for carrier-depletion-based silicon optical modulators," J. Lightw. Technol., vol. 30, no. 11, pp. 1602-1609, Jun. 2012.

[14] A. Samani et al., "Experimental parametric study of 128 Gb/s PAM-4 transmission system using a multi-electrode silicon photonic Mach Zehnder modulator," Opt. Express 25, 13252-13262 (2017).

[15] L. Breyne et al., "50 GBd PAM4 transmitter with a 55nm SiGe BiCMOS driver and silicon photonic segmented MZM," Optics Express, vol. 28, no. 16, pp. 23 950-23 960, 2020

[16] L. Breyne et al., "Electro-Optic Frequency Response Shaping using Embedded FIR Filters in Slow-Wave Modulators," Journal of Lightwave Technology, vol. 39, no. 6, pp. 1777-1784, 2020.

[17] D. Patel, M. Parvizi, N. Ben-Hamida, C. Rolland, and D. V. Plant, "Frequency response of dual-drive silicon photonic modulators with coupling between electrodes," Opt. Express, vol. 26, no. 7, pp. 8904-8915, Apr. 2018.

[18] P. Absil et al., "Reliable $50 \mathrm{~Gb} / \mathrm{s}$ silicon photonics platform for next generation data center optical interconnects," in Proc. IEEE Int. Electron. Devices Meeting, 2017, pp. 34.2.1-34.2.4.

[19] D. Patel et al., "Design, analysis, and transmission system performance of a $41 \mathrm{GHz}$ silicon photonic modulator," Opt. Express, vol. 23, no. 11, pp. 14 263-14 287, Jun. 2015.

[20] G. L. Li, T. G. B. Mason, and P. K. L. Yu, "Analysis of segmented traveling-wave optical modulators," J. Lightw. Technol., vol. 22, no. 7, pp. 1789-1796, 2004. 